\title{
EVOLUCIÓN DE LAS PRINCIPALES VARIABLES DE ÁRBOLES DE Pinus taeda L. SOMETIDOS A DIFERENTES TRATAMIENTOS SILVICULTURALES EN EL NORDESTE DE LA PROVINCIA DE CORRIENTES, ARGENTINA ${ }^{1}$
}

\author{
EVOLUTION OF THE MAIN VARIABLES OF Pinus taeda L. TREES UNDER DIFFERENT \\ SILVICULTURAL TREATMENTS IN NORTHEASTERN CORRIENTES PROVINCE, ARGENTINA
}

\author{
Hugo E. Fassola ${ }^{1}$ Fabio A. Moscovich ${ }^{1}$ Paula Ferrere $^{1}$ Francisco Rodríguez A. $^{2}$
}

\section{RESUMEN}

El presente estudio fue desarrollado con el propósito de cuantificar las relaciones existentes entre raleos, podas y crecimiento tanto en plantaciones, como en árboles de Pinus taeda L., y con la finalidad de construir modelos que puedan predecir la producción y calidad de productos futuros. Se propuso como objetivo analizar la información de las variables diámetro a la altura del pecho (dap), altura total y volumen cilindrométrico $\left(\mathrm{dap}^{2}{ }^{*} h\right)$ en un ensayo, instalado en el año 1996 en las cercanías de la localidad de Santo Tomé, Provincia de Corrientes (Argentina). Para cumplir con el objetivo propuesto se generaron mediante raleo selectivo a los 3 años cuatro densidades diferentes (1666; 833; 416 y 208 pla/ha). En cada densidad se aplicaron cuatro intensidades de podas $(0 ; 30 ; 50$ y 70\%, respecto de la profundidad de copa verde), las cuales se efectuaron en 2, 3 y 4 realces con intervalos de un año entre podas. Una vez analizados los datos obtenidos, después de 5 años de observación, se puede concluir que las variables analizadas se vieron afectadas, en distintos grados, por la intensidad de los raleos y por los grados y número de podas aplicados. La altura total fue la variable menos afectada, aunque las podas fuertes (mayores a 50\% de remoción de copa verde) alteraron negativamente su evolución. La práctica de raleos fue efectiva cuando se realizaron podas del 30\% trabajando en densidades bajas (416 plantas por hectárea) debido a que los ejemplares lograron igual crecimiento que los tratamientos sin poda.

Palabras-claves: podas, raleos, incrementos, Pinus taeda.

\section{ABSTRACT}

A study was developed in order to examine the relationship between thinning, pruning and increment of trees and stands of Pinus taeda L., as well as to determine models that could predict production and quality of logs. Information on diameter at breath height (dbh), total height (h) and volume $\left(d b h^{2}{ }_{*} h\right)$ were obtained in a trial established in 1996, close to St. Tome city in Corrientes Province (Argentina). A systematic design was employed; at age three years plots of the original plantation were thinned to 1666, 833, 416 and 208 stem/ha. In each density, pruning was applied with four intensities, 0, 30, 50 and $70 \%$ of green crown depth removal and with one year interval different pruning-lift were applied, 2,3, and 4 lifts. Five years old data were analyzed. After the analysis it was concluded that tree variables were affected in different grades by thinning, pruning and number of pruning lift applied. Height was the less affected variable, although high intensity pruning (more than $50 \%$ ) have a negative effect on height growth. Thinning practice was effective when done in stands of low densities with pruning up to $30 \%$ of green crown removal, growth of these trees was similar to those growing in the control plots.

Key words: pruning, thinning, increment, Pinus taeda.

\section{INTRODUCCIÓN}

La producción forestal primaria en sí misma puede ser un proceso altamente sofisticado (Paredes, 1994). De hecho cuando se llevan a cabo intervenciones silvícolas, como podas y raleos, se está dimensionando una escuadría. De acuerdo con la oportunidad e intensidad con que hayan sido aplicados estos tratamientos se maximizará algún grado de calidad, basado en la falta de ramas y dimensiones del rollizo, afectando los beneficios económicos.

1. Trabajo Financado parcialmente por el Proyeto Forestal de Desarrollo, SAGPYA-BIRF, Pia 26/98 "Manejo Intensivo de Pinus taeda".

2. Ingenieros Forestales, EEA INTA Montecarlo, AE 4, (3384) Montecarlo, Misiones, Argentina. hfasola@ceel.com.ar

3. Ingeniero Forestal, Bosques Del Plata S.A., Calle 186, Parcela 3, Barrio San Isidro, (3300) Posadas, Misiones, Argentina. frodriguez@cmpc.com.ar

Recebido para publicação em 07/11/2001 e aceito em 10/07/2002. 
Si bien ya existen algunos ensayos sobre poda y raleo en Pinus taeda, como ser los citados por Stöhr et al. (1987) y Seitz (1995) éstos fueron realizados en los primeros años de la plantación y efectuándose un solo levante y una sola intervención de raleo; no está evaluada la relación que puede encontrarse cuando se combinan, varias operaciones consecutivas de poda o realces y distintas intensidades de raleos.

Con el fin de conocer el efecto de estas prácticas sobre rodales y ejemplares de Pinus taeda L., se establecieron ensayos con el objetivo de analizar y establecer la incidencia de tratamientos silvícolas de poda y raleo sobre el crecimiento en área basal, diámetros sobre muñones resultantes y evolución de las principales variables de árboles dominantes de esta especie (Fassola et al., 1999a; Fassola et al., 1999b; Fassola et al., 1999c; Fassola et al., 1999d).

Si bien fue factible a través de estos ensayos determinar y cuantificar diferencias en el crecimiento en área basal de los rodales a causa de los tratamientos aplicados, como también su efecto sobre el incremento en diámetro, en altura y en tamaño de árboles dominantes, aun no pudo determinarse si tratamientos muy diversos de poda y raleo generaban respuestas similares en la evolución de las principales variables de árboles de Pinus taeda L.

Con ese objetivo fue analizada la información generada durante el período 1996-2000 de las variables diámetro a la altura del pecho, altura y tamaño del árbol en un ensayo de raleo y poda de Pinus taeda.

\section{MATERIALES Y MÉTODOS}

El ensayo se encuentra ubicado en una plantación de Pinus taeda L., origen Marion, situado entre las coordenadas geográficas $56^{\circ}$ Long. oeste y $28^{\circ} 20^{\prime}$ Lat. sur. en las cercanías de la localidad de Santo Tomé, Provincia de Corrientes (Argentina). El clima se caracteriza por poseer una temperatura media anual de $21^{\circ} \mathrm{C}$ con una mínima absoluta de $-2^{\circ} \mathrm{C}$. La precipitación media anual durante el período de observación fue de 1994 mm (INTA, 2000). El relieve es suavemente ondulado con pendientes inferiores al 15\%, siendo los suelos pertenecientes al gran grupo Kandiudalf (Fernández et al., 1996).

El rodal, donde se realizó el ensayo, fue implantado en el año 1993, con una densidad inicial de 1666 plantas/ha (pla/ha). La única tarea de preparación del suelo consistió en subsolado y fertilización sobre la línea de plantación.

\section{Diseño}

El diseño del ensayo fue de tipo sistemático clinal (Alder, 1980; Fassola et al., 1999a; Fassola et al., 1999b; Fassola et al., 1999c). Por medio de raleos selectivos a los 3 años se generaron cuatro fajas de distintas densidades, 1666, 833, 416 y 208 pla/ha. En cada una de ellas se realizó la poda de ramas vivas, en subfajas perpendiculares a las anteriores, usando cuatro intensidades de remoción: 0; 30; 50 y 70 \% respecto de la profundidad de copa verde. En estas subfajas, a su vez, la poda fue efectuada con dos, tres y cuatro realces o levantes de poda - elevaciones de la altura de poda -, con intervalos de un año entre las mismas, generándose de esta forma 32 tratamientos (Tabla 1), cada uno de los cuales tienen 5 repeticiones.

\section{Recolección de datos}

A partir de los 3 años de edad se midieron anualmente, en todas las parcelas, los diámetros a la altura del pecho (dap) de todos los ejemplares.

Mediante muestreo al azar, dentro de cada parcela, se seleccionaron 2 ejemplares, a los cuales se les midió la altura total (h) y la base de copa verde (bcv).

\section{Procesamiento de la información}

Mediante el Sistema de Procesamiento de Datos de Parcelas Experimentales e Inventario (Keck et al., 1999) fue posible procesar la información de los árboles que fueron seleccionados al azar de cada repetición resultante de las mediciones del período 1996-2000. La información de los árboles de cada repetición correspondiente a tratamientos similares fue unificada, resultando en un total de 10 observaciones por año/tratamiento. 
TABLA 1: Tratamientos de raleos y podas aplicados a Pinus taeda L.

TABLE 1: Thinning and pruning treatments applied to Pinus taeda L.

\begin{tabular}{ccc|c|c|c|c|c|c|c|c}
\hline Tratamiento & $\begin{array}{c}\text { Densidad } \\
(\mathrm{pla} / \mathrm{ha})\end{array}$ & $\begin{array}{c}\text { Raleo }^{1} \\
(\%)\end{array}$ & $\begin{array}{c}\text { Poda }^{2} \\
(\%)\end{array}$ & $\begin{array}{c}\text { Realces }^{3} \\
\left(\mathrm{n}^{\mathrm{o}}\right)\end{array}$ & Tratamiento $^{\text {Densidad }}$ & $\begin{array}{c}\text { Raleo }^{1} \\
(\mathrm{pla} / \mathrm{ha})\end{array}$ & $\begin{array}{c}\text { Poda }^{2} \\
(\%)\end{array}$ & $\begin{array}{c}\text { Realces }^{3} \\
\left(\mathrm{n}^{\circ}\right)\end{array}$ \\
\hline 1 & 1666 & 0 & 0 & 0 & 17 & 416 & 75 & 0 & 0 \\
2 & 1666 & 0 & 30 & 2 & 18 & 416 & 75 & 30 & 2 \\
3 & 1666 & 0 & 30 & 3 & 19 & 416 & 75 & 30 & 3 \\
4 & 1666 & 0 & 30 & 4 & 20 & 416 & 75 & 30 & 4 \\
5 & 1666 & 0 & 50 & 2 & 21 & 416 & 75 & 50 & 2 \\
6 & 1666 & 0 & 50 & 3 & 22 & 416 & 75 & 50 & 3 \\
7 & 1666 & 0 & 50 & 4 & 23 & 416 & 75 & 50 & 4 \\
8 & 1666 & 0 & 70 & 2 & 24 & 208 & 87,5 & 0 & 0 \\
9 & 1666 & 0 & 70 & 3 & 25 & 208 & 87,5 & 30 & 2 \\
10 & 833 & 50 & 0 & 0 & 26 & 208 & 87,5 & 30 & 3 \\
11 & 833 & 50 & 30 & 2 & 27 & 208 & 87,5 & 30 & 4 \\
12 & 833 & 50 & 30 & 3 & 28 & 208 & 87,5 & 50 & 2 \\
13 & 833 & 50 & 30 & 4 & 29 & 208 & 87,5 & 50 & 3 \\
14 & 833 & 50 & 50 & 2 & 30 & 208 & 87,5 & 50 & 4 \\
15 & 833 & 50 & 50 & 3 & 31 & 208 & 87,5 & 70 & 2 \\
16 & 833 & 50 & 50 & 4 & 32 & 208 & 87,5 & 70 \\
\hline
\end{tabular}

Em que: ${ }^{1}=\%$ de remoción del $n^{0}$ de plantas; ${ }^{2}=\%$ de remoción de copa viva; ${ }^{3}=$ número de elevaciones de la altura de poda, intervalo entre realces 1 año.

\section{Análisis estadístico}

Mediante análisis de regresión simple se procedió a ajustar modelos del tipo $y=\exp (a+b /$ edad $)$ para las variables dap, h y $\operatorname{dap}^{2} * h$ (volumen cilindrométrico) de cada tratamiento. Con posterioridad, mediante análisis de covarianza se realizó la comparación de los coeficientes correspondientes al intercepto (coeficiente $a$ ) y a la pendiente (coeficiente $b$ ) ajustados para cada tratamiento, de a pares, para determinar la existencia de grupos de tratamientos que no exhibieran diferencias significativas entre los mismos.

\section{RESULTADOS Y DISCUSIÓN}

\section{Evolución del diámetro a la altura del pecho (dap)}

En la Tabla 2, pueden observarse que los 32 tratamientos pudieron agruparse en 18 grupos, por no existir diferencias significativas, al $90 \%$ de probabilidad, entre los interceptos y las pendientes.

TABLA 2: Grupos homogéneos para la variable dap.

TABLE 2: Homogeneous groups for variable dbh.

\begin{tabular}{clll}
\hline Grupo & \multicolumn{1}{|c|}{ Tratamientos } & Grupo & \multicolumn{1}{c}{ Tratamientos } \\
\hline 1 & $1-12-13-31$ & 10 & $11-12-13-21-26-27-31$ \\
2 & $2-4-7-14-15-16-23-29-30-31$ & 11 & $12-13-21-30-31$ \\
3 & $3-4-7-14-15-30-31$ & 12 & $17-24-28$ \\
4 & $4-7-14-15-16-18-23-29-30$ & 13 & $18-19-20-21-26-27-28-31$ \\
5 & $5-7-15-30$ & 14 & $20-21-29-31$ \\
6 & $6-7-16-30-32$ & 15 & $21-22-27-28-29-31$ \\
7 & $7-8-14-15-16-22-23-29-30-32$ & 16 & $22-23-29-30-31$ \\
8 & $9-16-32$ & 17 & $23-29-30-31-32$ \\
9 & $10-17-18-19-21-28-31$ & 18 & $25-26$ \\
\hline
\end{tabular}

Del grupo 1 se desprende que el tratamiento 1, sin poda y sin raleo, tuvo una evolución diamétrica similar a la de tratamientos con intensidad de raleo del $50 \%$ y podas del $30 \%$ con 3 y 4 realces (tratamientos 12 y 13) o tratamientos con un raleo del $87,5 \%$ de la densidad original y podados al $70 \%$ con dos realces 
(T31).

Con estos resultados se puede inferir que la aplicación de un raleo a los 3 años, con una intensidad del $50 \%$, no permitió obtener ganancias diamétricas significativas respecto a los tratamientos con una intensidad de poda del $30 \%$, como también que podas intensas (70 \%) en bajas densidades (T31) atentarían contra el objetivo de maximizar el rendimiento en madera libre de nudos en pocos árboles.

Los grupos 2; 3 y 4 reflejaron que podas suaves, $30 \%$, realizadas en rodales con altas densidades (1666 pla/ha) generarían un crecimiento diamétrico de los árboles afectados, similar a la que se obtendría en densidades menores (208 pla/ha) y con podas más intensas (50-70\%).

Los grupos 7 y 8 permitieron inferir que altos niveles de poda, independientemente de la densidad del rodal, tuvieron patrones de evolución diamétrica similares.

En el grupo 9 pudo observarse que los tratamientos sin poda, para densidades de 833 y $416 \mathrm{pla} / \mathrm{ha}$, tuvieron una respuesta similar a la alcanzada con podas del 30 al $50 \%$ con distinto número de realces en la densidad de 416 pla/ha.

Tampoco los tratamientos sin poda, con densidades de 416 y 208 pla/ha, evidenciaron diferencias significativas en la evolución del dap, tal cual surge de la observación del grupo 12; esto refleja que los raleos efectuados por debajo de $416 \mathrm{pla} / \mathrm{ha}$, antes de los 7 años, generaron espaciamientos que fueron indiferentes para el desarrollo diamétrico de los ejemplares del rodal.

Un comportamiento similar exhiben los grupos 13,14,15 y 16, donde podas con intensidades del 30 $\%$ y $50 \%$, con 2 y 3 realces, tuvieron un comportamiento aproximadamente similar a los tratamientos con densidades de 416 y 208 pla/ha.

Analizando la Figura 1 se puede corroborar la evolución de la variable dap durante el período 19962000.
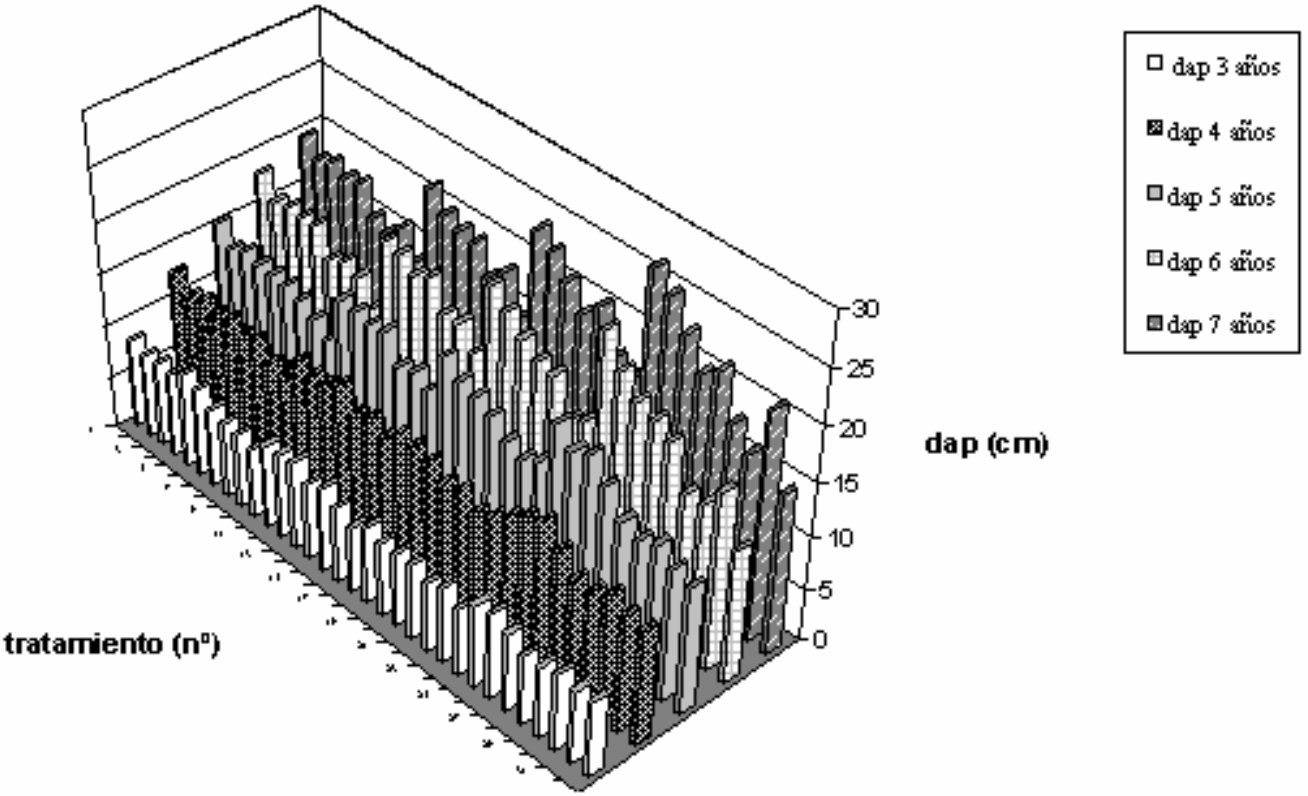

ap (cm)

FIGURA 1: Evolución del dap de árboles de Pinus taeda L. en rodales sometidos a podas y raleos.

FIGURE 1: Dbh evolution of Pinus taeda L. trees in pruned and thinned stands.

Este resultado es coincidente con lo encontrado por Stöhr et al. (1987) quien encontró diferencias en la evolución del diámetro y el volumen cuando comparaba individuos fuertemente podados (60\% de remoción de copa respecto a la altura total) con el testigo (individuos sin podar) a los cuatro años de haber aplicado el tratamiento, con una sola poda y a los cuatro años de aplicación del tratamiento.

Seitz (1995), destaca que los incrementos en diámetro muestran una tendencia parabólica, cuya mayor inclinación se encuentra cuando se realiza la remoción del $0 \%$ al $40 \%$ de la altura total del árbol; por lo tanto, podas con mayor intensidad no representan ganancias importantes en el crecimiento del diámetro; 
cuando se realiza una sola intervención.

\section{Evolución de la altura (h)}

En la Tabla 3 puede observarse que los 32 tratamientos consiguen agruparse en 17 grupos por no existir diferencias significativas, al $90 \%$ de probabilidad, entre los interceptos $(a)$ y las pendientes $(b)$.

Los grupos 1, 2 y 3 se caracterizan por incluir en ellos los tratamientos sin poda correspondientes a las densidades 1666, 833 y 208 pla/ha, podas en esas mismas densidades y en la densidad de 416 pla/ha, de intensidades del $30 \%$ y hasta 4 realces y sólo incluir intensidades de poda del $50 \%$ en los tratamientos 5, 6 y 21, aunque estos últimos en no más de 3 realces.

Los grupos 6 y 7, por su parte, incluyen tratamientos de todas las densidades con intensidades de poda del 50 \% en 2, 3 y 4 realces (trat. 16, 22, 28 y 29) y del 70 \% con 2 y 3 realces (trat. 8,9 y 31).

Estos agrupamientos indicarían, en primera instancia, que las densidades de los rodales empleados en el ensayo y las intensidades de poda no superiores al $30 \%$ no afectaron mayormente el desarrollo en altura. Observando la Figura 2 puede afirmarse que las mayores causas de afectación de la evolución en altura fueron la intensidad y número de realces de poda.

TABLA 3: Grupos homogéneos para la variable altura (h). TABLE 3: Homogeneous groups for the variable height (h).

\begin{tabular}{c|lcll}
\hline Grupos & \multicolumn{1}{|c}{ Tratamientos } & Grupos & \multicolumn{1}{c}{ Tratamientos } \\
\hline 1 & $1-2-3-4-5-10-11-12-20-21-24-25-26-27$ & 10 & $12-17-20-21-23-24-25-26-27-29-30-31$ \\
2 & $2-3-4-5-6-10-11-12-13-21-24-25-26$ & 11 & $13-19-21-25-26-27$ \\
3 & $3-4-5-6-11-12-13-21-25-26-27$ & 12 & $14-20-21-25-26-27-31$ \\
4 & $5-6-11-12-13-20-21-25-26-27-31$ & 13 & $15-16-17-20-21-22-23-24-25-27-28-29-30-$ \\
5 & $7-14-15-16-17-20-21-22-24-27-28-29-30-31$ & 14 & $16-20-21-22-23-24-27-28-29-30-31-32$ \\
6 & $8-16-22-28-29-31$ & 15 & $17-19-20-21-22-25-27-28$ \\
7 & $9-16-22-28-29-31$ & 16 & $18-19-20-21-24-28$ \\
8 & $10-11-12-17-19-20-21-24-25-31$ & 17 & $20-21-22-23-24-25-26-27-28-29-30-31$ \\
9 & $11-12-13-17-19-20-21-24-25-26-27-30-31$ & & \\
\hline
\end{tabular}

Efectuado el ajuste de modelos de la evolución de la altura para los tratamientos del grupo 1 y del grupo 6 (por ser considerados grupos extremos), pudo observarse el fuerte diferencial en altura para ambos grupos a los 7 años de edad (Figura 3). Y de mantenerse las pendientes establecidas, los tratamientos con mayor intensidad de poda (grupo 6) verían afectada negativamente su evolución en altura y consecuentemente la incorporación de nuevo follaje en el plano vertical, con las consecuencias lógicas de atraso en el crecimiento.

Por otra parte, considerando que la muestra fue tomada al azar, es dable pensar que la altura dominante se vea también afectada, ya que al evaluar la misma se observó un comportamiento similar al encontrado por Fassola et al. (1999c), con lo cual al evaluar sitio, por medio de la misma, se podría incurrir en errores si los modelos no recogen las variaciones en el patrón de desarrollo que puedan inducir tratamientos extremos de poda. 


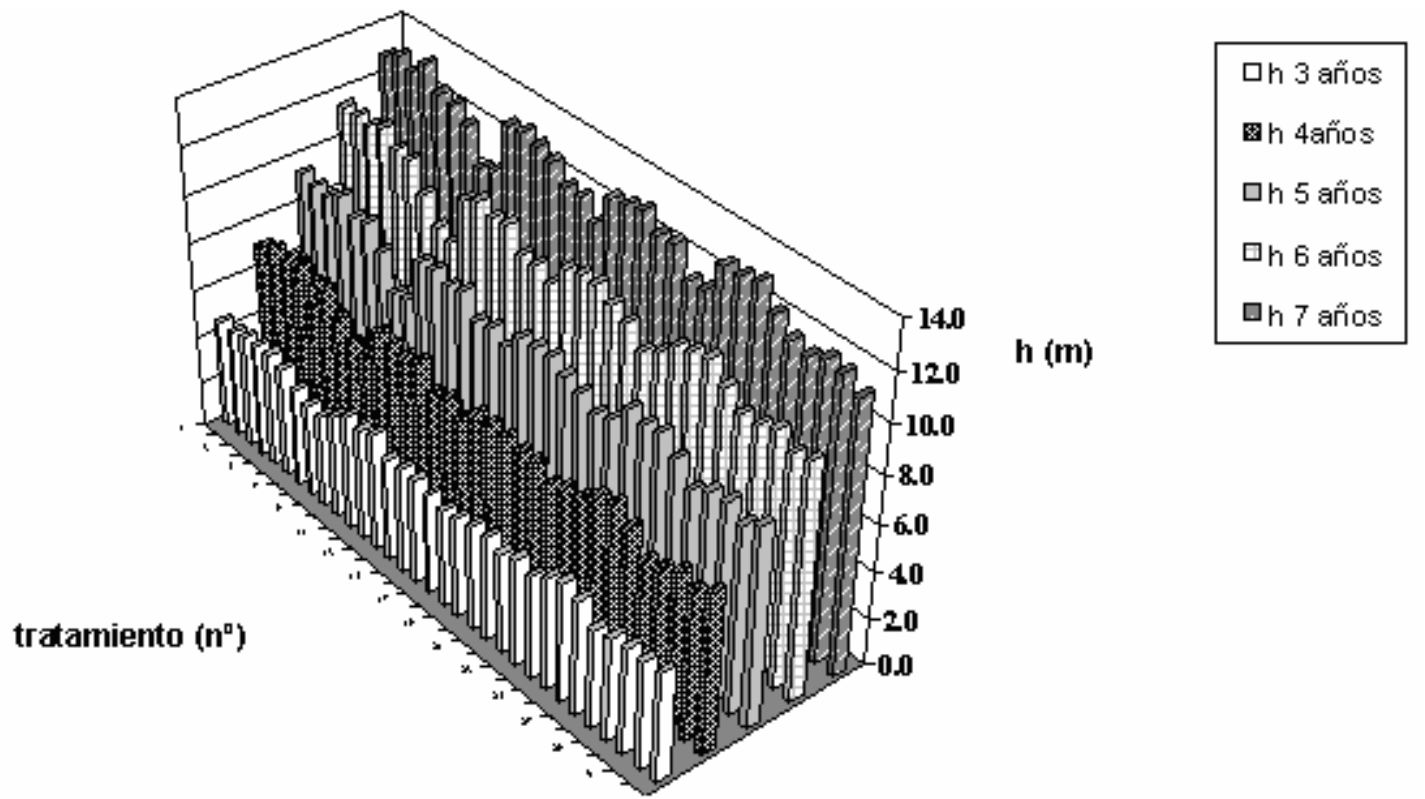

FIGURA 2: Evolución de la altura de árboles de Pinus taeda L. en rodales sometidos a podas y raleos. FIGURE 2: Height evolution of Pinus taeda L trees in pruned and thinned stands.

Aunque algunos tratamientos de podas suaves (30\%) exhiben al $7^{\circ}$ año una leve superioridad con relación al testigo sin poda de la misma densidad, indicando cierta recuperación, esto no se observó en los tratamientos con podas más intensas. Pero considerando la fuerte influencia de la edad en la tasa de incremento en altura, a mayor edad es menos probable que las diferencias con los tratamientos sin poda o con menor intensidad de desrame se reduzcan.

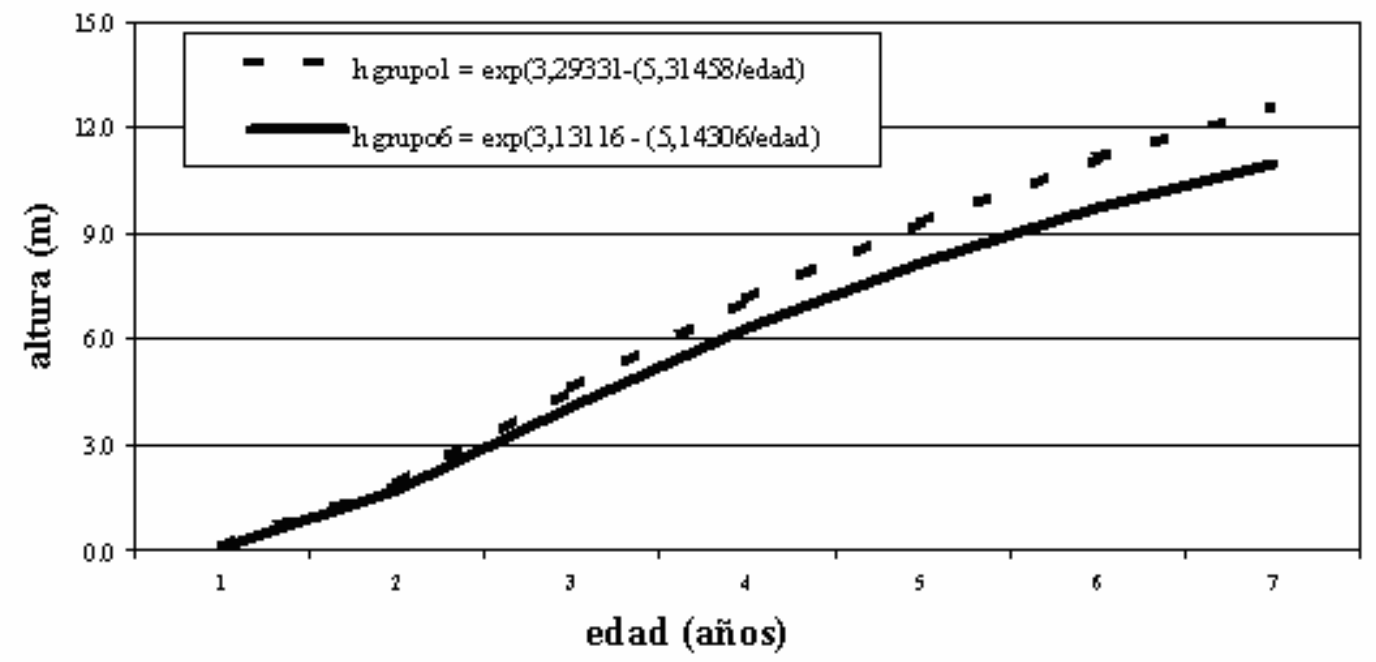

FIGURA 3: Evolución de la altura de árboles de Pinus taeda L. correspondientes a los grupos 1 y 6. FIGURE 3: Height evolution of Pinus taeda L trees of the groups 1 and 6.

Stöhr et al. (1987) no encontraron diferencias significativas cuando evaluaron la altura total de los individuos. Seitz (1995), también coincide en no haber encontrado diferencias significativas cuando evalúa incrementos en altura total de los individuos. En ambos casos, es de aclarar, que se trataba de una sola intervención de poda.

Si bien en los grupos anteriores no se observó fuertes agrupamientos por densidad, Crechi et al. (1992) establecieron diferencias significativas en el desarrollo en altura debido a distintas densidades iniciales de plantación en Pinus taeda L. En el caso bajo estudio del efecto de la densidad resultante de los raleos al $3^{\text {er }}$ año sobre la altura quedó evidenciado por los grupos 8 al 14, donde se agrupan distintos tratamientos de las densidades de 833, 416 y 208 pla/ha y los grupos 15 al 17 donde no hay diferencias entre 
tratamientos correspondientes a densidades de 416 y 208 pla/ha.

El efecto del sitio y la densidad del rodal sobre la relación altura-diámetro en rodales coetáneos ha sido tratado ampliamente (Prodan et al., 1997), sugiriéndose el empleo de modelos paramétricos que incluyan, además del diámetro, variables independientes como el número de árboles o la semisuma de la altura del árbol más grueso y del más delgado de la parcela, para describir la misma. Los resultados del presente ensayo inducen también a considerar la necesidad de contemplar la inclusión, en modelos para predecir la altura, de la intensidad de poda y el número de realces, sobre todo a partir de intensidades de poda superiores al 50 \% de remoción de la copa viva.

\section{Evolución del volumen cilindrométrico $\left(d a p^{2} * h\right)$}

En la Tabla 4 puede observarse que los 32 tratamientos pudieron concentrarse en 15 grupos, por no existir diferencias significativas al $90 \%$ de probabilidad entre los interceptos $(a)$ y las pendientes $(b)$.

El tamaño de los árboles alcanzado en cada tratamiento evidenció claramente el efecto de los raleos, intensidades de poda y número de realces sobre ellos, al afectar el dap, la h o ambas variables a la vez (Figura 4).

TABLA 4: Grupos homogéneos para la variable $d a p^{2}{ }_{*} h$.

TABLE 4: Homogeneous groups for the variable $d b h^{2}{ }^{*} h$.

\begin{tabular}{clcl}
\hline \multicolumn{1}{c|}{ Tratamientos } & Grupos & Tratamientos \\
\hline Grupos & \multicolumn{1}{c}{ 1-21-31 } & 9 & $11-21-27-31$ \\
2 & $2-3-4-15-21-31$ & 10 & $12-13-21-31$ \\
3 & $3-4-5-15-31$ & 11 & $15-25-29-30-31$ \\
4 & $5-6-13-15$ & 12 & $17-23-24$ \\
5 & $6-30-7$ & 13 & $20-21-28-30-31$ \\
6 & $7-8-14-15-16-22-23-29-30-31-32$ & 14 & $21-22-27-28-29-31$ \\
7 & $8-9-16-22-29-30-31-32$ & 15 & $25-26$ \\
8 & $10-17-18-19-21-25-28-31$ & & \\
\hline
\end{tabular}

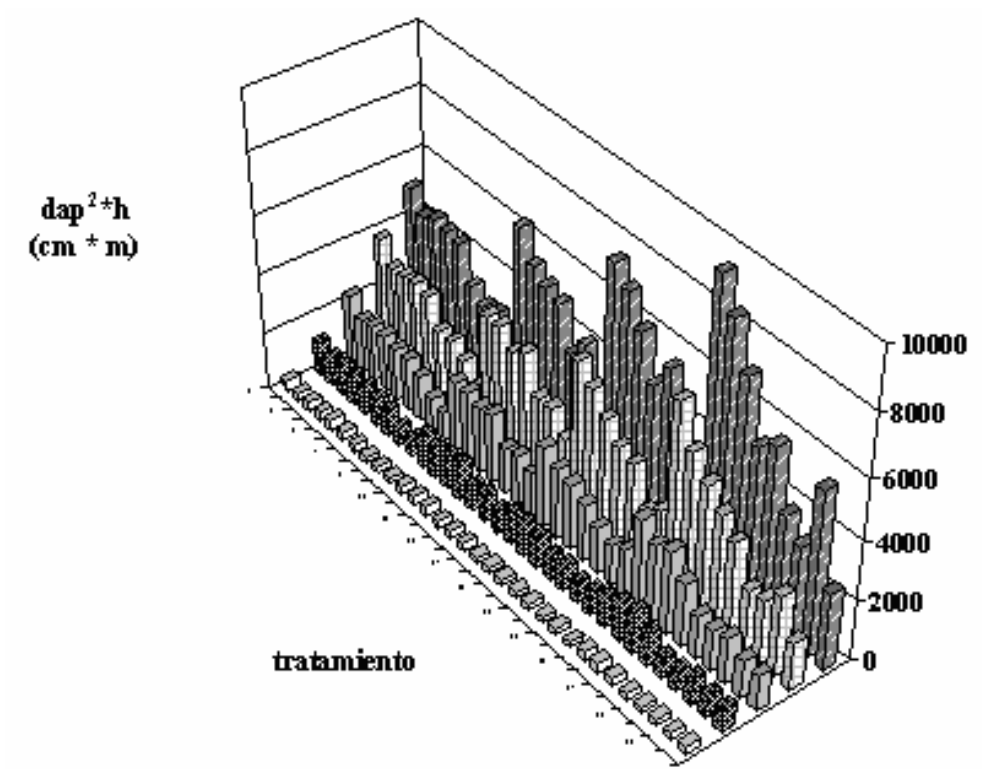

FIGURA 4: Evolución del volumen cilindromérico $\left(\operatorname{dap}^{2}{ }_{*} h\right)$ de árboles de Pinus taeda en rodales sometidos a podas y raleos.

FIGURE 4: Cylindrical volume $\left(d b h^{2}{ }^{*} h\right)$ evolution of Pinus taeda L trees in pruned and thinned stands. 
La modificación del área fotosintética del rodal por raleos y podas introdujo condiciones de competencia que arrojaron como resultado que árboles en rodales con altas densidades y sin raleo (trat. 1) alcanzaron desarrollos similares a los de ejemplares de rodales con menores densidades (tratamientos 21 y 31 con 416 y 208 pla/ha respectivamente) pero con fuertes remociones de copa viva (50 \% y 70 \%, ambos con dos realces) (Ver grupo 1).

Este comportamiento estaría reflejando niveles de “ocupación” (García, 1990) del sitio similares, por parte de ejemplares bajo muy distintas condiciones de crecimiento, estando fuertemente determinada la “ocupación” por la arquitectura de la copa.

En el caso de los grupos 6 y 7, el hecho de no existir diferencias significativas entre el tamaño de árboles que han crecido bajo muy distintas condiciones de densidad reflejó claramente el impacto de la remoción de copa viva del 50 \% o superior sobre las posibilidades de empleo de nuevos recursos generados por raleos.

El grupo 8 puso de manifiesto que podas del $30 \%$, con dos y tres levantes, en rodales con densidades de 416 pla/ha (trat. 18-19), permitieran a estos alcanzar desarrollos similares a los de los tratamientos 10 y 17, sin poda; reflejando que ese nivel de remoción de copa permitió generar condiciones de ocupación del sito por parte de los ejemplares tratados, semejantes a las de los que no recibieron podas dentro de ese grupo.

Tratamientos como raleos o podas fuertes generan condiciones donde los árboles residuales o tratados, no están en condiciones de hacer empleo inmediato de los recursos adicionales disponibles a causa de la remoción de los competidores o previamente deben recuperar su copa completamente (García, 1990), tal como se ha visto al analizar la evolución de variables como el dap, h o dap ${ }^{2} * h$.

Patiño et al. (2001) al analizar muestras de densidad de raíces en el perfil del suelo, tomadas de árboles de distintos tratamientos en este mismo ensayo a los 6 años de edad, establecieron la falta de diferencias significativas en la distribución de la misma para la mayor parte de las variantes silvícolas analizadas, aunque se evidenció cierta tendencia a un mayor desarrollo radicular en las menores densidades (Figura 5). Esto pondría en evidencia lo referido anteriormente en cuanto a la imposibilidad de hacer empleo inmediato de los recursos disponibles por parte del sistema radicular debido a la remoción de los competidores y que dentro de ciertos niveles en una gran gama de tratamientos los árboles harían una “ocupación” similar del sitio.

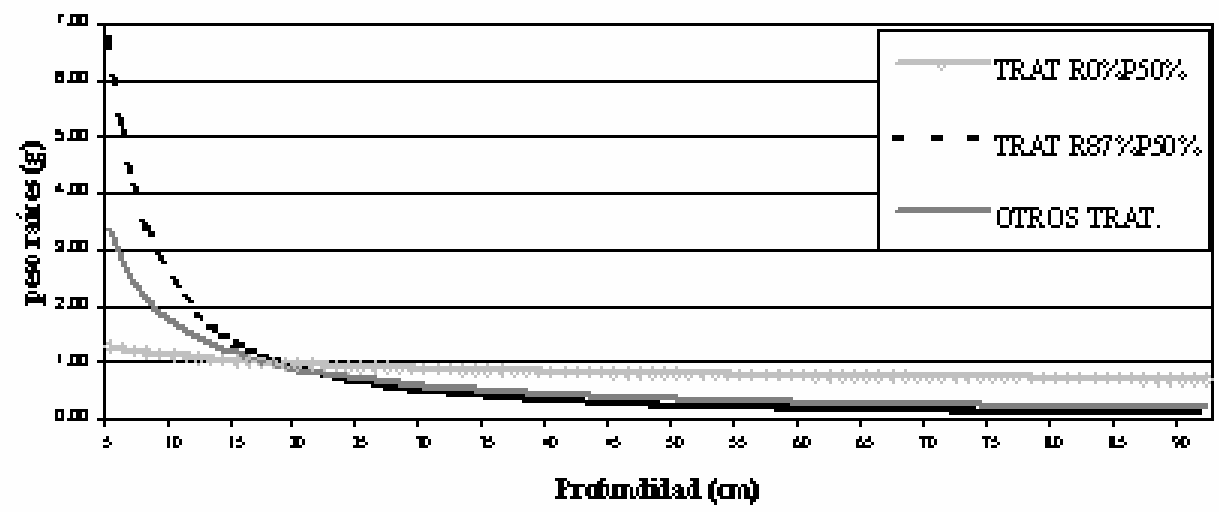

FIGURA 5: Distribución en el perfil del suelo del peso seco de raíces de árboles de Pinus taeda L. en rodales sometidos a podas (P) y raleos (R) (Fuente: Patiño et al., 2001).

FIGURE 5: Distribution of roots dry weight of Pinus taeda $\mathrm{L}$ trees in the soil profile in pruned (P) and thinned (R) stands (Patiño et al., 2001).

Procesos racionales de toma de decisiones requieren de la posibilidad de predicción de la respuesta a una amplia variedad de tratamientos silvícolas, algunos con efectos de mayor complejidad como la poda y que de no tenerse en cuenta pueden generar sesgos en la proyección de crecimientos mediante métodos tradicionales. Esa disminución de la capacidad de ocupación del sitio puede ser abordada o modelada a nivel rodal mediante variables de estado que representen el estado de "cierre" o nivel de uso de los recursos del 
mismo (García, 1990).

El empleo de la longitud de copa (García, 1990; Knowles et al., 1986), como variable que expresa las condiciones de uso de los recursos disponibles por parte de un rodal, ha sido una alternativa ya empleada para modelar el crecimiento de rodales jóvenes de Pinus taeda L. (Fassola et al., 1999b) o para explicar la disponibilidad forrajera bajo el dosel de esa especie (Fassola et al., 2001).

Sin embargo, tal como sostiene García (1990), este enfoque aparte de requerir una base de datos sólida, considera o trata de similar manera el follaje removido por raleos que el extraído de las partes inferiores de la canopia por podas, como también considera que la reducción de copa /ha es proporcional al porcentaje de árboles removido en vez de serlo al porcentaje del área basal o volumen y que la recuperación de la misma después del raleo o poda es igual a la tasa de crecimiento en altura.

\section{CONCLUSIONES Y RECOMENDACIONES}

Variables como el dap, la altura y el volumen cilindrométrico de árboles de Pinus taeda L. se han visto afectadas en distinto grado por la intensidad de los raleos y de las podas, éstas últimas también han afectado la evolución de estas variables en razón de la cantidad de realces aplicados; este resultado es coincidente con lo encontrado por Stöhr et al. (1987) quien encontró diferencias en la evolución del diámetro y el volumen cuando comparaba individuos fuertemente podados ( $60 \%$ de remoción de copa respecto a la altura total) con el testigo (individuos sin podar) a los cuatro años de haber aplicado el tratamiento.

Estos mismos autores no encontraron diferencias significativas cuando evaluaron la altura total de los individuos.

Seitz (1995), destaca que los incrementos en diámetro muestran una tendencia parabólica, cuya mayor inclinación se encuentra cuando se realiza la remoción del 0\% al 40\% de la altura total del árbol; por lo tanto, podas con mayor intensidad no representan ganancias importantes en el crecimiento del diámetro.

Seitz (1995), también coincide en no haber encontrado diferencias significativas, al igual que Stöhr et al (1987) cuando evalúa incrementos en altura total de los individuos.

Si bien la altura fue, proporcionalmente, la variable menos afectada, los tratamientos de poda intensos, generalmente superiores al $50 \%$, han promovido fuertes alteraciones en su evolución, las cuales deberán considerase al momento de modelar su desarrollo.

El volumen cilindrométrico de los árboles, alcanzado entre los tres y siete años, también fue afectado por raleos y podas, pudiendo establecerse que niveles de poda del $50 \%$ o superiores no son recomendables, ni aun aplicando raleos.

La práctica de raleos fue efectiva en podas del 30 \% de remoción de copa viva para una densidad de $416 \mathrm{pla} / \mathrm{ha}$ ya que los ejemplares alcanzaron una evolución similar a la de los tratamientos sin poda de una densidad de $416 \mathrm{pla} / \mathrm{ha} \mathrm{y} 833 \mathrm{pla} / \mathrm{ha}$.

Los tratamientos de poda y raleos generaron situaciones de “ocupación” individual del sitio similar para tratamientos en apariencia disímiles, por lo que se recomienda la inclusión de variables que contemplen el grado de ocupación con el propósito de ajustar modelos de predicción que permitan evaluar adecuadamente regímenes en condiciones de rodal densos o abiertos, como también sometidos a podas enérgicas.

\section{AGRADECIMIENTOS}

Se agradece a Papelera del Plata S.A. la posibilidad de haber podido realizar este ensayo, como también a sus técnicos Ing. Ftales. Raúl Pezzutti y Manuel Durán.

Se agradece también la colaboración del Ing. Ftal. Diego Allegranza, quien participó en los estadios iniciales del estudio, como del Ing. Ftal. Arturo Hernández, por su cooperación en las mediciones efectuadas y de los Ings. Agr. Simón Kramer y Oscar J. Burtnik de la AER INTA Sto. Tomé, por el apoyo brindado.

\section{BIBLIOGRAFÍA}

ALDER, D. Estimación del volumen forestal y predicción del rendimiento. Roma: FAO, 1980. v.2. (Montes 22/2). 
CRECHI, H.E.; FRIEDL, R.A.; FERNÁNDEZ, R.A. Efecto de la densidad de plantación sobre el crecimiento de Pinus taeda L. Eldorado: Jornadas Sobre Pinos Subtropicales, 1992. p.92-110. (CIEF, 7).

FASSOLA, H.E.; RODRÍGUEZ, F.; ALLEGRANZA, D.; et al. Resultados iniciales de tratamientos silvícolas directos en Pinus taeda origen Marion, en el NE de Corrientes. Montecarlo: INTA EEA, 1999a. 9p. (Informe Técnico n.22).

FASSOLA, H.E.; RODRÍGUEZ, F.; FERRERE, P.; et al. Crecimiento de árboles dominantes en fase juvenil de Pinus taeda $L$ en el NE de Corrientes sometidos a distintos tratamientos silvícolas. Montecarlo: INTA EEA, 1999b.19p. (PIA 20/96.Informe final).

FASSOLA, H.E.; FERRERE, P.; RUIBAL DE GELID, M. Crecimiento en área basal de plantaciones jóvenes de Pinus taeda L. origen Marion. Montecarlo: INTA EEA, 1999c. 11p. (PIA 20/96. Informe final).

FASSOLA, H.E.; FERRERE, P.; RODRÍGUEZ, A.F. Predicción del diámetro sobre muñones en árboles podados de Pinus taeda L. origen Marion en el NE de Corrientes. Montecarlo: INTA EEA, 1999d. 9p. (PIA 20/96. Informe final).

FASSOLA, H.E.; FERRERE, P.; LACORTE, S.M.; et al. Predicción de la producción de un pastizal bajo distintas estructuras de canopia de Pinus taeda L. origen Marion en el NE de Corrientes, Argentina. Montecarlo: INTA EEA, 2001. 38 p.

FERNÁNDEZ, R.; PAHR, N.; LUPI, A. Evaluación del crecimiento de Eucalyptus grandis Hill ex Maiden en diferentes condiciones de sitio del nordeste argentino. Aguas de Lindoia (SP): 1996. CD.

GARCÍA, O. Growth of thinned and pruned stands. In: James, R.; Tarlton, G.L. (Eds.) New approaches to spacing and thinning in Plantation Forestry: Proceedings of an IUFRO Symposium, Rotorua, New Zeland, 10-14 April 1989. New Zeland: Ministry of Forestry, 1990. p.84-97. (FRI Bulletin n.151).

INTA. Registros pluviométricos. Corrientes: AER Santo Tome, 2000.

KECK, A.; CRECHI, E.; FASSOLA, H.E.; et al. Sistema de procesamiento de datos de parcelas experimentales e inventario. Montecarlo: INTA EEA, 1999. Version 1.0.

KNOWLES, R.L.; WEST, G.G. The use of crown length to predict the effects of pruning and thinning in Pinus radiata. In: FUJIMORY, T.; IBARAKI, D. (Ed.) Crown and canopy structure in relation to productivity. (Japón): Forestry and Forest products Research Institute. 1986. p.104-117.

PATIÑO, C.; FASSOLA, H.E.; FERRERE, P. Informe de densidad de raíces de Pinus taeda L. origen Marion sometido a distintos tratamientos silvícolas en el perfil del suelo, a los 7 años de edad. Inédito. Montecarlo: CECOAL - INTA EEA, 2001. 2p. (Montecarlo - Papelera del Plata).

PAREDES, V. Como se adaptará la industria forestal y de la madera a la incorporación de Chile: acuerdos de libre comercio con países vecinos. Industria Forestal y Madera, Santiago, Chile, 1994.

PRODAN, M.; PETERS, R.; COX, F.; et al. Mensura forestal. (CATIE : Série Investigación y Educación en Desarrollo Sostenible). IICA-BMZ-GTZ, 1997. p.329-330.

SEITZ, R.A. Manual da poda de espécies arbóreas florestais. Curitiba: FUPEF/DURATEX S.A./KLABIN Fabricadora de Papel e Celulose, 1995. 88p.

STÖHR, G.; EMERECIANO, D. B.; FABER, J. Green pruning of Pinus taeda and its influence on growth in Parana Brazil. Actas: Simpósio sobre silvicultura y mejoramiento genético de especies forestales. Buenos Aires: CIEF, 1997. t.4, p.197-204. 